\title{
Controlling Chaos in A Dual-Ring Erbium-Doped Fiber Laser by Modulating The Pump
}

\author{
Sen-Lin YAN ${ }^{1, a}$ \\ ${ }^{1}$ School of electronic engineering, Nanjing XiaoZhuang University, Nanjing, 20017, China \\ asenlinyan@163.com
}

Keywords: Control, Erbium-Doped Fiber Laser, Chaos, Cycle.

\begin{abstract}
Control of chaos in a dual-ring erbium-doped fiber laser is studied by modulating the laser pump and its physical model is presented to induce the dual-ring erbium-doped fiber laser to a cyclic state and other dynamics states via adding a periodic signal to the pump. When one pump is modulated by a periodic square wave signal, we find that chaotic behaviors of the laser can be pressured and the dual-ring can show two periodic states while each ring can emit cyclic pulses. It indicates that chaotic dual-ring erbium-doped fiber laser has been controlled. When another periodic square wave signal with a different modulation depth value is used to perform on the laser, behavior of the laser can be deduced to produce multi-periodic states. When the pump is modulated by a sinusoidal signal, behaviors of the laser can be controlled to deduce two other periodic states while each ring emits cyclic pulses. We find that chaotic behavior of the laser can be controlled. And the laser shows a lot of dynamics behaviors via the chaos-control method. The result is very helpful to study of chaos-control techniques, fiber lasers and other lasers.
\end{abstract}

\section{Introduction}

Chaos or its motion is very sensitive to its starting condition. Chaotic behavior or chaos output signal shows a kind of random characteristic. Chaotic system and its applications were widespread studied by many researchers. Since "OGY" technology of chaos-control was presented in twentieth Century 90[1], many chaos-control methods have been presented and educe a chaotic variety to a periodic regular variety [2,3]. Newly, study of chaos-control is focal point. By using the application of a small perturbation or occasional proportional feedback, we can convert chaotic behavior into a stable state or one of the unstable periodic orbits embedded in a chaotic attractor. It has been recently obtained that chaotic lasers can be realized to suppress or stabilized by the current modulation and the optical feedback, and so on[4]. However, few reports of chaos-control in dual-ring erbium-doped fiber lasers are presented. In this paper, a novel method of chaos-control is presented using a periodic signal to modulating the each pump. The results are very helpful to our study of chaos-control techniques, dual-ring erbium-doped fiber lasers, and other lasers.

In twentieth Century 90, erbium doped fiber as a novel of laser source and optical amplifier has been used in optical fiber communication systems and optical fiber sensors. People made widely studies of erbium-doped fiber laser system and its dynamics in theorey and experiment. Because of the metastable state lifetime of erbium ion in fiber laser for $1 \sim 10 \mathrm{~ms}$ and the optical fiber core of high power density, laser can be made by the continuous working state transition to self pulsation or chaotic state, F.Sancheez and L.G.Luo et al observed that the nonlinear dynamics phenomena, and established the model of two coupled lasers [5,6]. It showes for potential value of application of chaos in secure communication and control fields, therefore, it is significance for researches on behavios and chaos-control in optical fiber laser. At present, double ring fiber laser can show chaos through nonlinear coupling, single ring fiber laser arises chaos through optical mutual injection of the two lasers [7,8]. The form of pulse fiber lasers are mainly self pulsation, where the nonlinear effect of strong irregular pulsation produced by the optical fiber lasers coupling. When high erbium ion doping concentration reaches a certain number of the saturated absorption, fiber laser can also produce irregular pulse or chaos[7,8]. 


\section{Model and Results}

The physical model for single ring erbium doped fiber laser may be a three level system. Because of the pump, Erbium ions transfer fast from the level 3 to level 2, so the activation level 3 remained almost vacancy. The physical model of single ring erbium doped fiber laser can be simplified as a two level system. The physical model for dual-ring erbium doped fiber laser may be described by coupling the two level systems. The normalized ion number density in the level 2 of the energy excited state and the cavity photon number density are described by the coupling rate equation as following $[7,8]$

$$
\begin{gathered}
\frac{d}{d t} E_{a}=-k_{a}\left(E_{a}+\eta_{0} E_{b}\right)+g_{a} E_{a} D_{a} \\
\frac{d}{d t} E_{b}=-k_{b}\left(E_{b}-\eta_{0} E_{a}\right)+g_{b} E_{b} D_{b} \\
\frac{d}{d t} D_{a}=-\left[1+I_{p a} \times\left[1+\delta_{a} \times S_{a}\left(2 \pi f_{a} t\right)\right]+\left|E_{a}\right|^{2}\right\} D_{a}+I_{p a} \times\left[1+\delta_{a} \times S_{a}\left(2 \pi f_{a} t\right)\right]-1 \\
\frac{d}{d t} D_{b}=-\left[1+I_{p b}+\left|E_{b}\right|^{2}\right\} D_{b}+I_{p b}-1
\end{gathered}
$$

Where subscripts $a$ and $b$ stand for a ring and $b$ ring of the lasers. Ip is the optical pump. E is the laser normalized field describing the total ion population density in each ring. $\mathrm{D}$ is the normalized ion number density. $\mathrm{k}$ is the loss coefficient of the laser. $\eta_{0}$ is the coupling coefficient of optical coupler. $\mathrm{g}$ is the laser gain coefficient. Chaotic optical pulses and other nonlinear dynamic behaviors in the laser can generate due to the dual-ring coupling. And in Eqs.(3), expression $\left[1+\delta_{a} \times S_{a}\left(2 \pi f_{a t}\right)\right]$ is the modulation items. $\delta_{\mathrm{a}} \times \mathrm{S}_{\mathrm{a}}\left(2 \pi \mathrm{f}_{\mathrm{a}} \mathrm{t}\right)$ as an additional freedom of operation is introduced by modulating the pump of the ring a. S stands for a periodic signal, $\delta$ is the modulation depth and $\mathrm{f}$ is the modulation frequency. When an additional freedom of pump modulation is induced to perform on the laser, we will find that the laser output shows cyclic pulses or other pulses. Control of chaos in dual-ring erbium-doped fiber lasers are realized by modulating the laser pump. Fig.1 shows our chaos-control scheme.

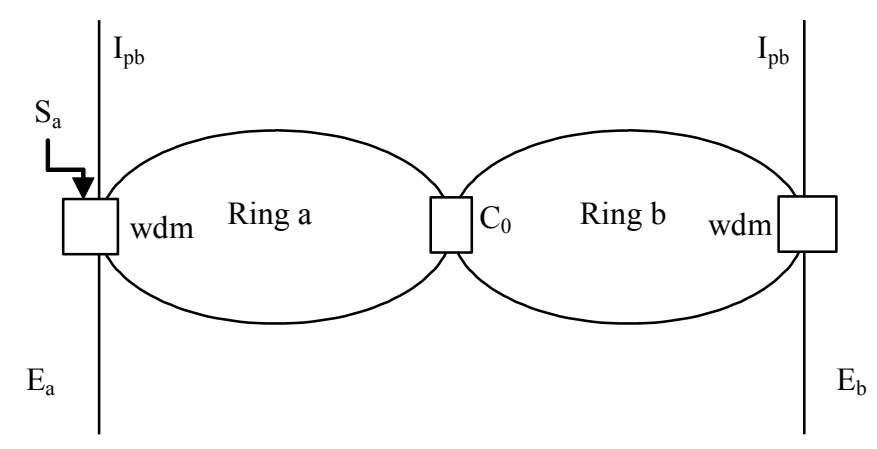

Fig.1 Chaos-control scheme. Where $\mathrm{I}_{\mathrm{pa}}$ and $\mathrm{I}_{\mathrm{pb}}$ are pump light, $\mathrm{C}_{0}$ is the coupler, wdm is the wavelength division multiplexer, $\mathrm{E}_{\mathrm{a}}$ and $\mathrm{E}_{\mathrm{b}}$ are the lasing fields output from ring a and ring $b$, respectively. $\mathrm{S}_{\mathrm{a}}$ and $\mathrm{S} b$ are the modulation signals.

The dual-ring erbium doped fiber lasers can generate chaotic dynamic behaviors due to the dual-ring coupling. In our numerical simulations, the laser system parameters are taken by normalized values [7,8]: $\mathrm{I}_{\mathrm{pa}}=\mathrm{I}_{\mathrm{pb}}=4, \mathrm{k}_{\mathrm{a}}=\mathrm{k}_{\mathrm{b}}=1000, \mathrm{\eta}_{0}=0.2, \mathrm{~g}_{\mathrm{a}}=4800, \mathrm{~g}_{\mathrm{b}}=10500$. Figure 2 shows no control of the dual-ring erbium-doped fiber laser nonlinear dynamic behavior, fig.2 (a) and (b) are 
chaotic attractor and dual-ring output optical fields. Chaos output signals have random characteristic and chaotic attractor and its trajectory have ergodic property in limit phase space. And its motion is very sensitive to its starting condition because chaotic trajectory is not the same all over time when the initial value is taken as different values.

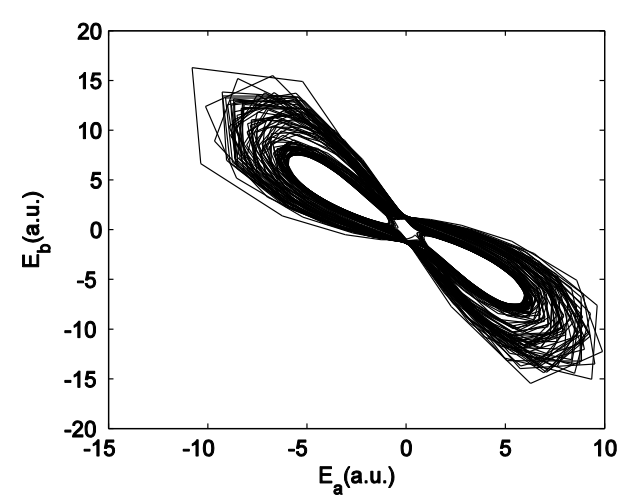

(a) Chaotic attractor
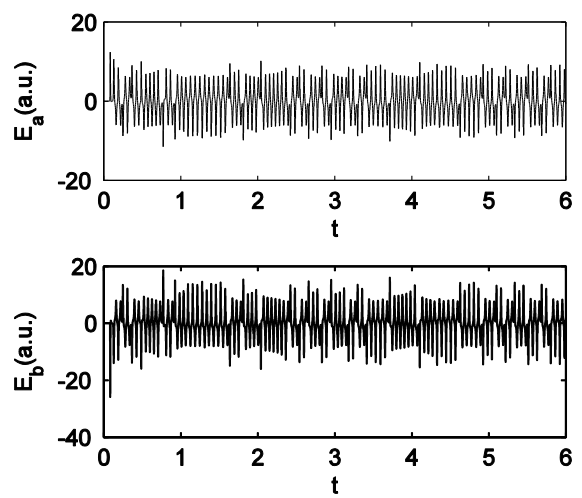

(b) Waveform

Fig.2 Chaos dynamic behavior

Firstly, we give results about control of the ring a via a square wave modulating the pump. At first, we take the control parameters $\delta_{\mathrm{a}}=0.05, \mathrm{f}_{\mathrm{a}}=18 \mathrm{MHz}$ and the square wave amplitude being 1 . We perform on modulation-control after $2 \mathrm{~ms}$ for all control. Chaos-control result is shown in Figs.3. We find that chaotic laser has been controlled to stabilize in a cycle-4 state after $5 \mathrm{~ms}$. Figure 3 (a) shows the laser dynamics being a cycle-4 orbit, fig.3 (b) shows dual-ring output waveform variation while the laser shows some undamped relaxation oscillation with the control-performance before 5ms, and fig.3 (c) shows two ring output in two cycle-4 states. The results indicate that chaos-control of the dual-ring erbium-doped fiber laser has realized to stabilize in cycle states.

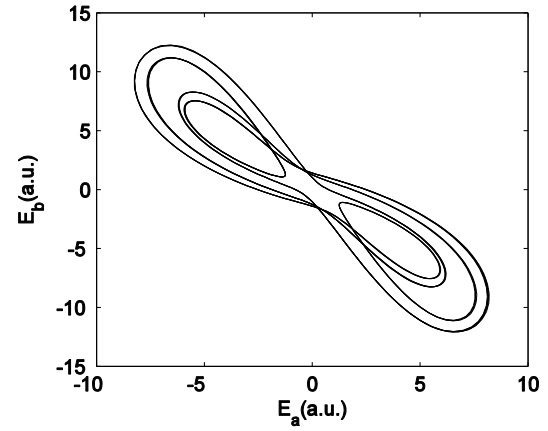

(a) Cycle-4
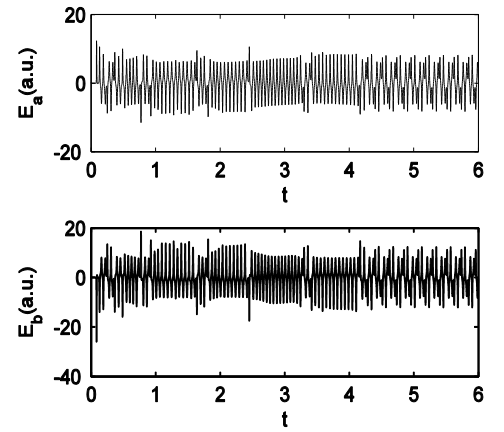

(b) Two ring output behaviors
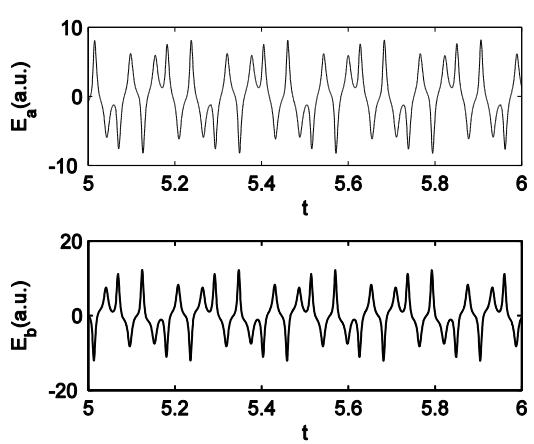

(c) Two cycle-4 waveforms

Fig. 3 The laser into cycle-4 states

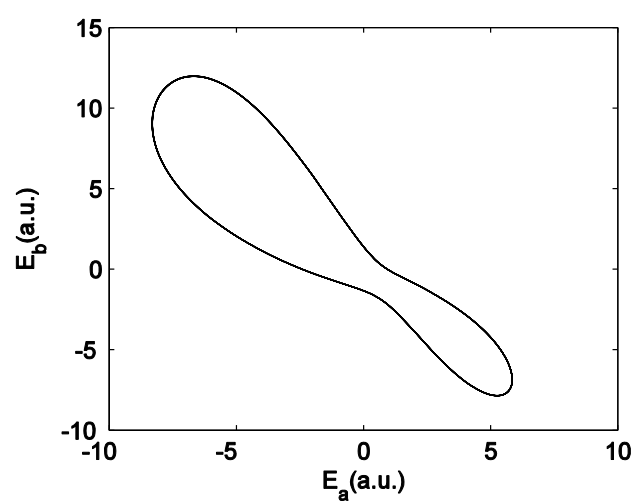

(a) A single-cycle orbit
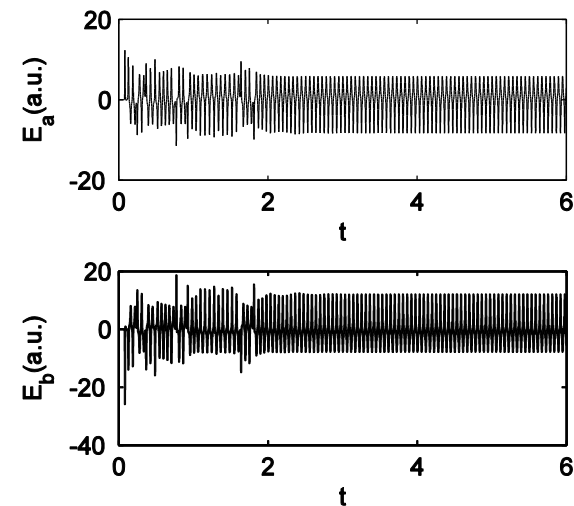

(b) Two ring output behaviors

Fig.4 The laser into single-cycle states 
Second, we give a single-cycle state result when the control parameters are taken as $\delta_{\mathrm{a}}=0.08$, $\mathrm{f}_{\mathrm{a}}=18 \mathrm{MHz}$ and the square wave amplitude being 1 . Figure 4 shows that the laser has been controlled to deduce to single-cycle states. Two ring of the laser emit cycle pulses after $2.5 \mathrm{~ms}$. Figure 4 (a) shows the laser dynamics behaviors presenting a single-cycle orbit, and fig.4 (b) shows two ring output waveforms and two ring output becoming of two single-cycle states after $2.5 \mathrm{~ms}$. The results indicate that chaos-control of the dual-ring erbium-doped fiber laser has obtained to pressure in cycle states.

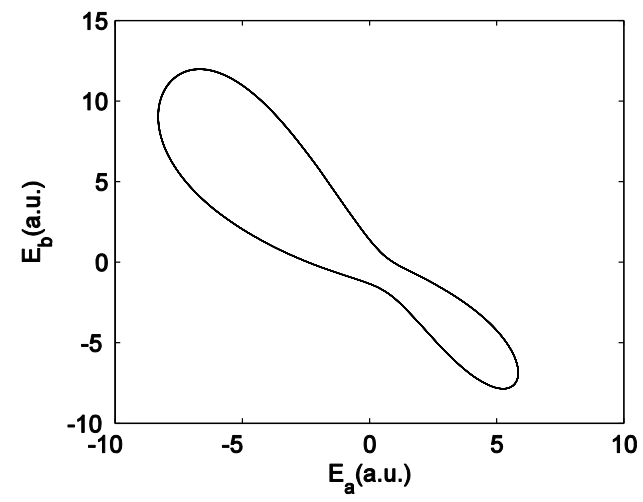

(a) another single-cycle orbit
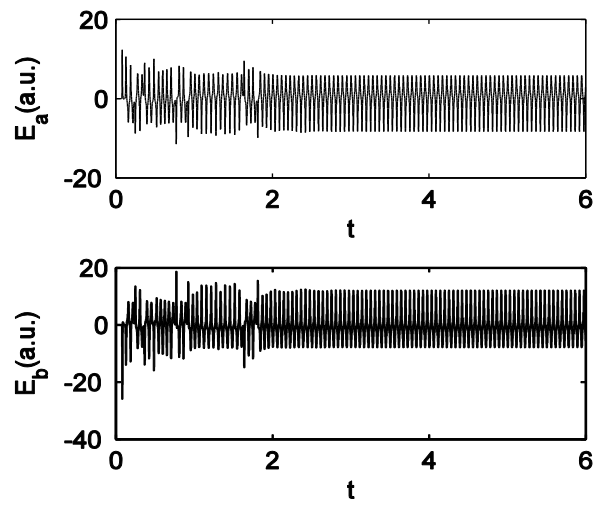

(b) Two ring output behaviors

Fig.5 The laser into other single-cycle states

Next, we give results via a sinusoidal signal modulating the pump. The control parameters are taken as $\delta_{\mathrm{a}}=0.1, \mathrm{f}_{\mathrm{a}}=18 \mathrm{MHz}$ and the amplitude being 1 . Chaos-control result is presented in Figs.5. We find that chaotic laser has been deduced to stabilize in a single-cycle state after $3 \mathrm{~ms}$. Figure 5 (a) shows the laser dynamics becoming of a single-cycle orbit. Figure 5 (b) shows dual-ring output waveform variation while the laser shows some undamped relaxation oscillation with the control-performance between $2 \mathrm{~ms}$ and $3 \mathrm{~ms}$. The results indicate that chaos-control of the dual-ring erbium-doped fiber laser has realized to educe single-cycle states.

\section{Summary}

We present a method of chaos-control of dual-ring erbium-doped fiber laser and its physical model. By numerical study of chaos-control processes, we find that chaos-control of the dual-ring erbium-doped fiber laser has realized. The laser can be controlled to deduce single-cycle states or other multiple-cycle states. The method is very helpful to study of chaos-control techniques, dual-ring erbium-doped fiber lasers, and other lasers.

\section{References}

[1] E .Ott, C. Grebogi , J. A. Yorke, Controlling chaos, Phys.Rev.Lett. 64(1990) 1196-1199.

[2] D. Ming, K. C. Peter, Analysis of Er-doped fiber laser stability by suppressing relaxation oscillation, IEEE J.Photonic Technology Letters 8 (1996) 1151-1153.

[3] S.L. Yan, Study on the method of controlling chaos in an Er-doprd fober dual-ring laser via external optical injection and shifting optical feedback light, Chaos 17 (2007),013106- 06.

[4] S.L.Yan, Control of chaos in an injection multi-quantum-well laser via shifting or modulating the injection light, J. Mod. Opt. 56 (2009) 539- 547.

[5] O. L. Williams, J. G. Ojalvo, and R. Roy, Fast intercavity polarization dynamics of an erbium-doped fiber ring laser: inclusion of stochastic effects, Phys.Rev.A 55 (1997) 2376-2385.

[6] F. Sancheez, G. Stephan, General analysis of instabilities in erbium-doped fiber laser, Phys.Rev.E 53 (1996) 2110-2122. 
[7] L. G. Luo, P. L. Chu, H. F. Liu, 1-GHz optical communication system using chaos in erbium-doped fiber lasers, IEEE J. Quantum Electron. 12(2000):269.271.

[8] R. Wang, K. Shen, Synchronization of chaotic systems modulated by another chaotic system in an erbium-doped fiber dual-ring laser system, IEEE J. Quantum Electron. 37 (2001) 960-963. 